\title{
BMJ Mortality in carotid sinus Open hypersensitivity: a cohort study
}

\author{
Joanna L Hampton, ${ }^{1}$ Carol Brayne, ${ }^{2}$ Michelle Bradley, ${ }^{2}$ Rose Anne Kenny ${ }^{3}$
}

To cite: Hampton JL, Brayne C, Bradley M, et al. Mortality in carotid sinus hypersensitivity: a cohort study. BMJ Open 2011;1: e000020. doi:10.1136/ bmjopen-2010-000020

- Prepublication history for this paper is available online. To view these files please visit the journal online (http://bmjopen.bmj.com).

Received 10 November 2010 Accepted 6 April 2011

This final article is available for use under the terms of the Creative Commons Attribution Non-Commercial 2.0 Licence; see http://bmjopen.bmj.com

\footnotetext{
${ }^{1}$ Department of Geriatric Medicine, Addenbrookes Hospital, Cambridge, UK ${ }^{2}$ Department of Public Health and Primary Care, Forvie Site, Cambridge, UK ${ }^{3}$ Mercers Institute for Ageing and Trinity College Institute of Neurosciences, Trinity College, Dublin, Ireland
}

Correspondence to Dr Joanna L Hampton; jlhampton@doctors.org.uk

\section{ABSTRACT}

Objective: Carotid sinus hypersensitivity (CSH) is common in older people. The authors hypothesise that patients with CSH have a higher mortality than a geographically, age-matched older cohort.

Design: A retrospective cohort study compared to geographical and age-matched data from the Office of National Statistics.

Setting: Specialist clinic in tertiary centre.

Patients: 1504 patients with CSH were identified from a single syncope outpatient assessment service between 1990 and 2001.

Interventions: Vital status was confirmed, and death certificates were sought for all deceased patients up to 2003.

Main outcome measures: Kaplan-Meier survival curves were analysed within the cohort according to three different subtypes of CSH. Standardised mortality rates (SMRs) were determined using geographical and age-matched data from the Office of National Statistics.

Results: There was no difference between CSH patients and the general population in SMRs for all causes, or for cerebrovascular or cardiovascular deaths. There was no difference in survival between the three subtypes of $\mathrm{CSH}(p=0.2)$ within the study cohort.

Conclusion: $\mathrm{CSH}$ is not associated with a higher mortality than the general population, and there are no differences in mortality between the three subtypes of $\mathrm{CSH}$. This confirms earlier findings and reinforces the neutral effect of CSH on mortality.

\section{INTRODUCTION}

Carotid sinus hypersensitivity (CSH) is a disease of older people, with a prevalence of between $25 \%$ and $48 \%$ in patients referred to hospital for unexplained syncope, falls or dizziness. $^{1-3}$

Three subtypes of carotid sinus hypersensitivity have been described: cardioinhibitory (CICSH), diagnosed if carotid sinus massage (CSM) results in three or more seconds of asystole; vasodepressor (VDCSH), diagnosed if carotid sinus massage results in a drop in systolic blood pressure of $50 \mathrm{~mm} \mathrm{Hg}$ or more; and mixed CSH, diagnosed if both cardioinhibition and vasodepression are present.

\section{ARTICLE SUMMARY}

\section{Article focus}

- Mortality rates in patients with CSH compared with age and geographically matched controls.

- Mortality rates compared between the three CSH subtypes, vasodepressor, cardioinhibitory and mixed.

\section{Key messages}

- This is the largest known cohort of patients with $\mathrm{CSH}$ to date.

- This confirms previous findings by smaller studies.

- CSH has no effect on mortality.

Strengths and limitations of this study

- This is a retrospective study.

- The sample size is large.

- It was not possible to address morbidity, as there was no control group. The control group for mortality was taken from the Office of National Statistics.

Syncope accounts for $3 \%$ of attendances at Accident and Emergency departments and $1 \%$ of admissions to a general hospital in the USA. ${ }^{4}$ Falls account for approximately $10 \%$ of visits to the Accident and Emergency department and $6 \%$ of admissions to a general hospital. ${ }^{5}{ }^{6}$ Of those admitted to hospital following a fall, only about $50 \%$ are alive 1 year later. Accidents are the fifth leading cause of death in older people, and falls constitute two-thirds of these accidental deaths, ${ }^{7}$ and are the commonest cause of injury-related death in persons over 75 years of age. ${ }^{8}$ CSH has a high incidence of morbidity, as it may lead to syncope, falls and accidents: approximately one in 10 falls leads to serious injury such as hip fractures, other fractures, subdural haematoma, soft-tissue injury or head injury. ${ }^{5} 910$ There is also an association between CSH and dementia. ${ }^{11}$

Despite these associations with significant markers of frailty and morbidity, there is limited evidence to date describing the implications of the presence of CSH for older people in terms of mortality. 
Brignole et al followed up 262 patients identified over a 6-year period who presented with syncope and were found to have CSH as a cause of their symptoms. The follow-up period was between 2 and 8 years (mean $44 \pm$ 24 months). The control group was 55 patients who had unexplained syncope, matched for age and sex. The overall mortality rate for the CSH patients was 7.3 per 100 person-years, compared with 5.8 per 100 personyears in the control group. The mortality rate after 5 years was $34 \%$ in the $\mathrm{CSH}$ group, compared with $27 \%$ in the control group. The standardised mortality rate (SMR) of the general population with a similar age and sex distribution was 8 per 100 person-years. The mortality rate in the CSH group was not influenced by CSH subtype. ${ }^{12}$ Similar findings were reported by Sutton et al; the 5 -year mortality rate was $36 \%{ }^{13}$

Engel hypothesised that death may result from prolonged asystole often following severe vasodepressor syncope, or that prolonged asystole could indirectly trigger malignant tachyarrhythmias. ${ }^{14}$ This study aims to reassess the mortality rate in a larger consecutive cohort of patients with symptomatic CSH compared with age and geographically matched statistics from the Office of National Statistics.

\section{METHODOLOGY}

One thousand five hundred and four patients with CSH were identified retrospectively from a single Syncope Service in the North East of England over a 10-year period. The service assessed patients referred either by GPs or by hospital physicians and was an outpatient facility. All patients aged 50 or over, referred for investigation of syncope or falls routinely undergo CSM using phasic BP recording equipment (Finometer/Finapres) in addition to orthostatic BP measurements and a surface ECG. The diagnosis of CSH was confirmed by reviewing hard copies of blood pressure and heart-rate tracings. The vital status of each patient was checked on the hospital Patient Administration System PAS) and via contact with the patients' General Practitioners (GPs). The Office of National Statistics (ONS) records all deaths in the UK. Death certificates for deceased patients were obtained from the ONS. Recorded death-certification details included date of death, place of death, address of last known residence, and underlying cause of death. The cause of death was classified into ischaemic heart disease, cerebrovascular disease, other circulatory diseases (including hypertensive heart disease, valvular heart disease, cardiac arrhythmias, ill-defined heart disease) and other causes (non-cardiovascular/noncerebrovascular) using the International Code of Disease, Nineth Revision. ${ }^{15}$

Data for the control group were compiled from population statistics published by the ONS. ${ }^{16}$ Mortality tables were identified from the North East (combining data from Tyne and Wear and Northumberland) and matched to the study cohort for age, sex and year of death. Figures were extracted from the ONS tables for death due to all causes, ischaemic heart disease and cerebrovascular disease.

\section{Statistical analysis}

The ages of the groups of patients who were alive or dead were analysed at two points: (1) at the time of diagnosis of CSH and (2) on the date at which the patient was last identified. This was the age at death for the deceased patients, and the age when they were confirmed to be alive by the GP for the living patients (known as 'last known age').

Survival analysis between the three subtypes of CSH was tested by the Kaplan-Meier method. Kaplan-Meier survival curves represent the cumulative probability of an individual remaining free of an endpoint, in this case, death.

Cause of death was compared with mortality statistics from the ONS, which were matched for age, sex and geographical location. Standardised mortality rates for all-cause mortality and mortality due to ischaemic heart disease and cerebrovascular disease were calculated (see below). To ensure an accurate comparison with the data from the ONS, the number of deaths was stratified by age (divided into 5-year age-bands) and by the year in which the death occurred. It was not possible to further stratify by gender because of insufficient sample size within each age group and year. The total number of patients within the study cohort changed every year: the cohort was identified retrospectively, and thus all patients diagnosed as having CSH between 1990 and 31 December 2001 were included. Thus, patients were entering the cohort every year, but some patients were dying each year, and the length of follow-up varied according to the year of diagnosis. This meant the total number of patients within the cohort was different each year. In population statistics from the ONS, the population is calculated from a midyear estimate based on the latest census information and total number of deaths. In order to make a comparison between the study cohort and the general population of the North East, the number of person years observed (PYOs) within the study cohort for each year was calculated. Person years calculates the number of people living for each year, that is, the proportion of each year an individual is living after they entered the study calculated as a decimal of a year (eg, a person who entered the study on 01.01.1990 and died in June 1992, would have 1 PYO for 1990, 1 PYO for 1991, and 0.5 PYO for 1992). The total PYOs were calculated for the entire cohort $(\mathrm{n}=1504)$ and then stratified by 5 -year age-bands and years.

Total deaths for all cause mortality, IHD and CVD deaths in 1991-2003 were recorded for the general population from the North East, and also stratified by 5-year age bands. Crude death rates were then calculated for both the study population and the general population in the North East. The crude death rate in the study population is the total number of deaths divided by the number of PYOs for each age and year. The death rate for the study cohort is expressed as death rate per 1000 
PYOs (the crude rate multiplied by 1000). Similarly, the crude death rate in the general population is the total number of deaths divided by the number of people within the population for each age and year. The death rate for the general cohort is expressed as death rate per 1000 population at risk (again, the crude rate multiplied by 1000 ).

To account for the differing age structures within the two populations, the SMR was calculated. The indirect method of standardisation was used comparing the expected number of deaths calculated from the general population with the observed number of deaths in the study cohort. The expected number of deaths was calculated by using the age structure of the study group; the PYOs in the study cohort were multiplied by the crude death rate in the North East population. This figure was then rounded up to whole numbers.

A SMR of less than 1 suggests the study cohort has fewer deaths than the expected figure in the general population; a figure of greater than 1 suggests there are more deaths in the study cohort than the general population.

\section{RESULTS}

One thousand five hundred and four patients were initially identified from the Syncope Service with definite CSH (median age 76.7 years, $59 \%$ female). Six hundred and seventy-eight $(45 \%)$ patients were VDCSH, 427 (28\%) CICSH and 399 (26\%) mixed CSH.

Three hundred and forty-five patients $(23 \%)$ of the 1504 were recorded as deceased on the PAS. A further 240 deceased patients were identified by telephoning GPs, resulting in a total of $585(40 \%)$ dead and 910 $(60 \%)$ living patients. The vital status of nine patients was untraceable (figure 1). During the study period, a further 11 patients died and were included in subsequent mortality analysis. The date of death was untraceable in 10 patients: thus survival analysis was completed on 1485 patients $(99 \%)$. Survival rates for patients with CICSH, VDCSH and Mixed CSH were

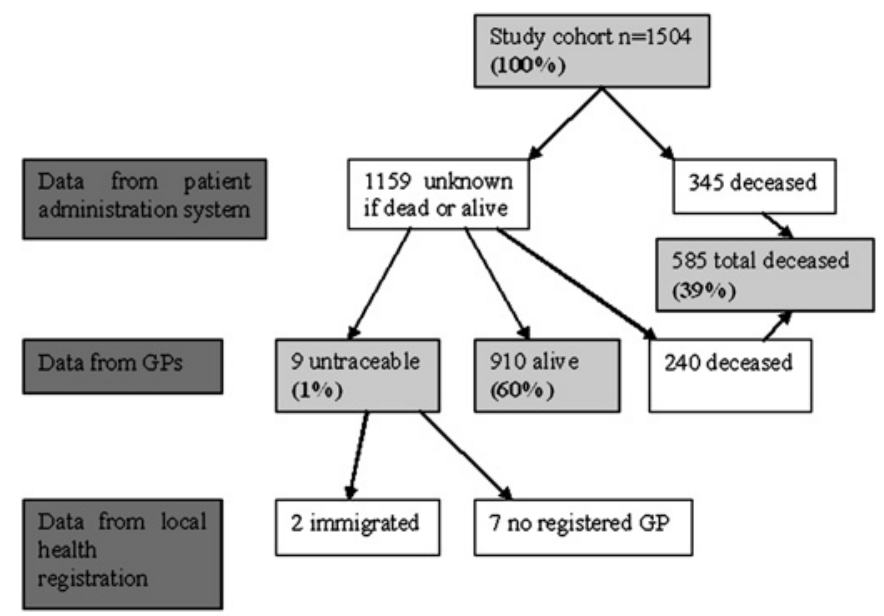

Figure 1 Vital status of the cohort at the beginning of study. similar $(\mathrm{p}=0.2)$. The median survival time for CICSH was 8 years (95\% CIs 7.3 to 8.7 years); the median survival time for VDCSH was 7 years (95\% CIs 6.4 to 7.4 years), and the median survival time for mixed $\mathrm{CSH}$ was 8 years (95\% CIs 7.4 to 8.6 years) (figure 2).

There was no difference between the numbers of deaths due to all causes in patients with CSH compared with the general population of the North East in any year with the exception of 2002 and 2003 (SMR 1.4 (95\% CI 1.1 to 1.7 ) and SMR 1.8 (95\% CI 1.1 to 2.9); table 1).

The year-on-year point estimates predictably suggest these ratios move from under one to over one over time as the cohort matures. The number of deaths in the study cohort and the control group increased with age for all causes, ischaemic heart disease (IHD) and cerebrovascular disease (CVD). There was no difference in any year between the numbers of deaths due to IHD or CVD in patients with CSH compared with the control group.

Death certificates were available for 581 of 596 requested (98\%). Death due to non-cardiovascular/noncerebrovascular causes (classified as 'other causes') was most common (324 patients $(56 \%)$ ). Of cardiovascular causes, 117 patients $(20.1 \%)$ died of ischaemic heart disease; 96 patients $(16.5 \%)$ died from stroke disease, and 44 patients $(7.6 \%)$ died from other circulatory diseases. Diseases of the respiratory system were the most common cause of death within the 'other causes' category, affecting 110 patients (19\%). Nineteen patients (3\%) were coded as dying from mental disease, and all were suffering from dementia. A further 16 patients (3\%) were coded as dying from 'symptoms, signs and illdefined conditions' and the death certificates in all these cases listed causes describing senescence such as 'old age' or 'frailty.'

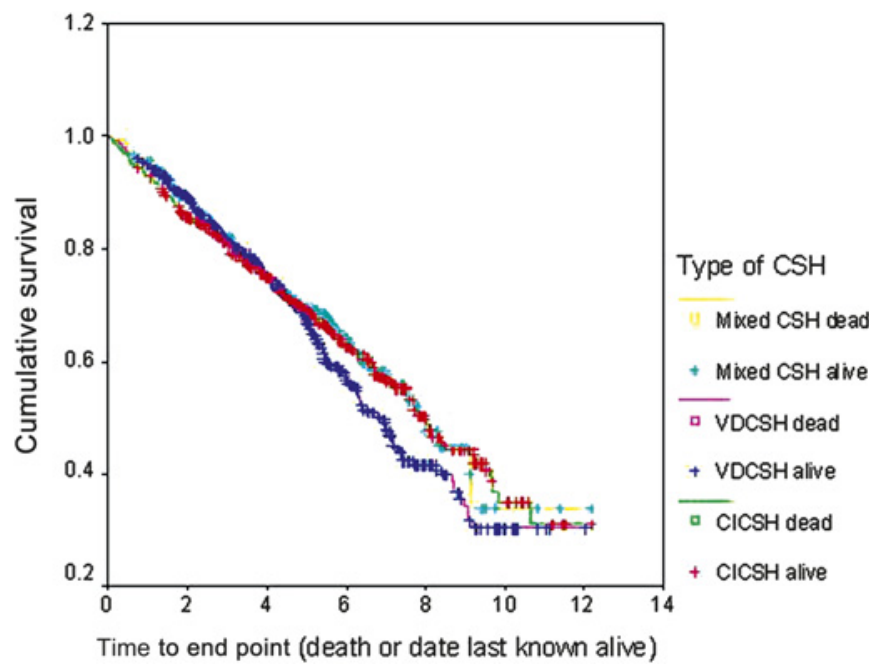

Figure 2 Kaplan-Meier survival curve for the study cohort comparing subtypes of carotid sinus hypersensitivity $(n=1485)$. $\mathrm{CICSH}$, cardioinhibitory carotid sinus hypersensitivity; $\mathrm{CSH}$, carotid sinus hypersensitivity; VDCSH, vasodepressor carotid sinus hypersensitivity. There is no significant difference in mortality between the three CSH subtypes. 


\begin{tabular}{ll}
$\begin{array}{l}\text { Table } 1 \\
\text { death }\end{array}$ & Standardised mortality ratios for all causes of \\
\hline Year & $\begin{array}{l}\text { Standardised mortality } \\
\text { ratio }(95 \% \mathrm{Cl})\end{array}$ \\
\hline 1991 & $0.5(0.01$ to 3.0$)$ \\
1992 & $1.4(0.6$ to 2.8$)$ \\
1993 & $1.0(0.5$ to 1.8$)$ \\
1994 & $0.5(0.3$ to 0.9$)$ \\
1995 & $0.7(0.5$ to 1.1$)$ \\
1996 & $0.7(0.5$ to 1.0$)$ \\
1997 & $0.8(0.6$ to 1.1$)$ \\
1998 & $1.1(0.9$ to 1.4$)$ \\
1999 & $1.0(0.8$ to 1.2$)$ \\
2000 & $1.2(0.9$ to 1.4$)$ \\
2001 & $1.2(0.9$ to 1.4$)$ \\
2002 & $1.4(1.1$ to 1.7$)$ \\
2003 & $1.8(1.1$ to 2.9$)$ \\
\hline
\end{tabular}

\section{DISCUSSION}

This paper has shown that, in a large cohort study of 1500 patients, mortality is similar to age and geographically matched controls. It confirms earlier findings from smaller cohorts published by Sutton $^{13}$ and Brignole. ${ }^{12}$ The methodologies for analysis of survival rates between the subtypes of $\mathrm{CSH}$ in this study and Brignole's study are similar, and Brignole has kindly given permission for the data from his study to be published in this paper for comparison (figure 3). Figure 2 from this study and figure 3 from Brignole's study show similar Kaplan-Meier curves for the three CSH subtypes and confirm that there is no difference in survival rates between CICSH, VDCSH and Mixed CSH.

In Brignole's control group, the morbidity (including rate of stroke, myocardial infarction and atrial fibrillation) over a 5-year follow-up period was comparable with that of the CSH group suggesting a neutral effect of CSH on morbidity. This finding could not be confirmed in our cohort, as there was no control group. However, more recent evidence also reinforces the possibility that for certain individuals, having CSH may be entirely benign: in 2006, a community-based study of individuals aged 65 and over who were asymptomatic for falls, syncope and dizziness found that CSH was prevalent in $35 \% .{ }^{17}$ This raised the possibility that $\mathrm{CSH}$ was not a pathological diagnosis but rather an age-related phenomenon. More recently, Tan $e t a l^{18}$ have also discussed this possibility following their findings that only $25 \%$ of patients (age range 38-98 years; mean age 71.5 years) presenting with classical symptoms for CSH (syncope, drop attacks and unexplained falls) were found to have CSH following CSM. This figure is lower than the figure observed in Kerr's study of asymptomatic community-dwelling patients over 65 and may be explained by referral bias and demographic differences but adds support for the hypothesis that CSH is an age-related physical entity rather than a causal mechanism for certain symptoms such as syncope, falls and drop attacks.

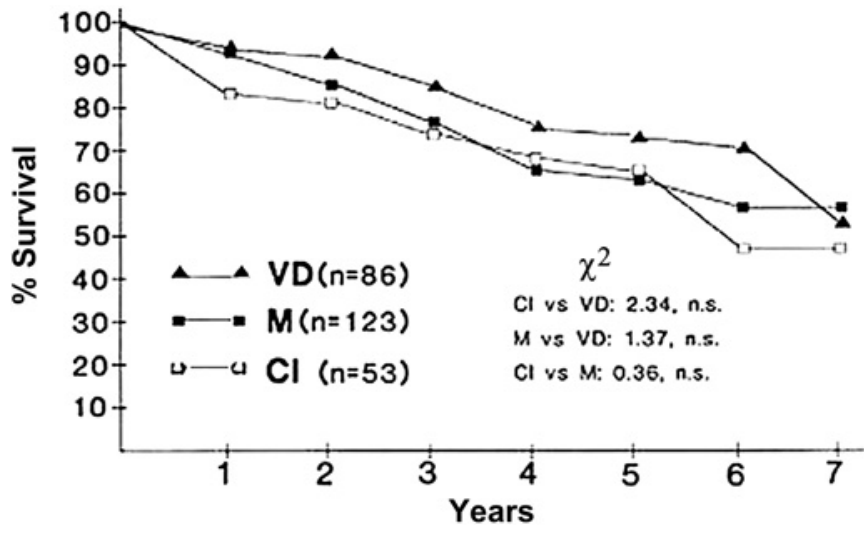

Figure 3 Predicted cumulative survival rates in patients with cardioinhibitory $(\mathrm{Cl})$, mixed $(\mathrm{M})$ and vasodepressor (VD) forms carotid sinus syndrome. The Kaplan-Meier curve is from Brignole et $a l^{12}$ (reproduced with the authors' permission).

If this is true, then an increase in mortality in patients with CSH would not be expected. The standardised mortality ratio of patients in the study cohort for death by all causes was increased in 2002 and 2003, but there was no increase from 1991 to 2001. This is likely to reflect small sample sizes rather than true clinical significance. It may also be a reflection of the method of mortality analysis and the comparative population.

The study cohort was generated from a specialist clinic in a teaching hospital. This may have introduced bias. Retrospective data collection also leads to further bias due to poorly recorded information that was not collected with clear questions in mind.

In summary, this paper confirms previous findings that there is no increase in mortality rates in patients with $\mathrm{CSH}$ compared with the general population. Despite this finding, it is important to remember that management goals for patients with CSH should remain the same: patients with CICSH and syncope in particular benefit from pacing, and this will add greatly to a reduction in symptoms and morbidity.

Acknowledgements Thanks to J Pairmen, research nurse at the Royal Victoria Infirmary.

Funding This research received no specific grant from any funding agency in the public, commercial or not-for profit sectors.

Competing interests None.

Contributors JH, RAK, CB and MB all contributed substantially to the study design and made significant contributions to the data acquisition and interpretation of data. All authors contributed to the manuscript, and all authors approved the final version.

Provenance and peer review Not commissioned; externally peer reviewed.

Data sharing statement All statistical calculations and an appendix detailing annual SMR are available from the corresponding author.

\section{REFERENCES}

1. Kenny RA, Traynor G. Carotid sinus syndrome-clinical characteristics in elderly patients. Age Ageing 1991;20:449-54.

2. Mclntosh SJ, Lawson J, Kenny RA. Clinical characteristics of vasodepressor, cardioinhibitory, and mixed carotid sinus syndrome in the elderly (see comments). Am J Med 1993;95:203-8. 
3. Allcock LM, O'shea D. Diagnostic yield and development of a neurocardiovascular investigation unit for older adults in a district hospital. J Gerontol A Biol Sci Med Sci 2000;55:M458-62.

4. Day SC, Cook EF, Funkenstein $\mathrm{H}$, et al. Evaluation and outcome of emergency room patients with transient loss of consciousness. Am J Med 1982;73:15-23.

5. Sattin RW. Falls among older persons: a public health perspective. Annu Rev Public Health 1992;13:489-508.

6. Runge JW. The cost of injury. Emerg Med Clin North Am 1993;11:241-53.

7. Rubenstein LZ. Syncope in the Older Patient. London: Chapman \& Hall, 1996.

8. Anon. HASS Listings for Men and Women Aged 50 and Above for Falls. London: Department of trade and industry, 1993.

9. Nevitt MC, Cummings SR, Hudes ES. Risk factors for injurious falls: a prospective study. J Gerontol 1991;46:M164-70.

10. Tinetti ME, Doucette J, Claus E, et al. Risk factors for serious injury during falls by older persons in the community. J Am Geriatr Soc 1995;43:1214-21.
11. Wentink JRM, Jansen RWMM, Hoefnagels WHL. The influence of age on the response of blood pressure and heart rate to carotid sinus massage in healthy volunteers. Cardiol Elderly 1993;1:453-9.

12. Brignole $\mathrm{M}$, Oddone $\mathrm{D}$, Cogorno $\mathrm{S}$, et al. Long-term outcome in symptomatic carotid sinus hypersensitivity. Am Heart J 1992;123:687-92.

13. Sutton R. Fourth European Symposium on Cardiac Pacing. Acta Stockholm 1989:133-6.

14. Engel GL. Psychologic stress, vasodepressor (vasovagal) syncope, and sudden death. Ann Intern Med 1978;89:403-12.

15. World Health Organization. International Classification of Diseases. 9th revision. Geneva: World Health Organization, 1977.

16. Office of National Statistics. http://www.statistics.gov.uk (accessed 2011).

17. Kerr SR, Pearce MS, Brayne C, et al. Carotid sinus hypersensitivity in asymptomatic older persons: implications for diagnosis of syncope and falls. Arch Intern Med 2006;166:515-20.

18. Tan MP, Newton JL, Reeve P, et al. Results of carotid sinus massage in a tertiary referral unit-is carotid sinus syndrome still relevant? Age Ageing 2009;38:680-6. 